\title{
Effect of Storage Period on Fenugreek Foliage Dehydrated by Different Methods
}

\author{
Janki Joshi* and Jatinderjit K. Gill \\ Department of Home Science, Surajmal Agarwal Private Kanya Mahavidyalaya, \\ Kichha-263148, Uttarakhand, India \\ Department of Family Resource Management, College of Home Science, PAU, \\ Ludhiana 141004, Punjab, India \\ *Corresponding author
}

\section{A B S T R A C T}

\section{Keywords}

Dehydration, Open sun drying, Solar drying, Microwave drying, Sensory evaluation

Article Info

Accepted:

07 October 2019

Available Online:

10 November 2019
Present research was undertaken to study the effect of storage period on fenugreek foliage dehydrated by different methods namely open sun drying without shade, open sun drying in shade, and solar drying without shade, solar drying with shade, microwave drying at $60 \%$ and at $80 \%$ power. The quality of fenugreek dehydrated by different methods and recipes prepared by using dehydrated product were compared immediately after dehydration and after two and four months of storage period in terms of colour, flavour, texture, taste and overall acceptability. It was observed that mean score for overall acceptability decreased with the storage period but changes were not significant. From the analysis of variance it was inferred that storage period had no significant influence on the overall acceptability of fenugreek dehydrated by different methods except open sun drying in shade. It was found that during the storage period of four months fenugreek dehydrated by microwave and solar drying with shade, solar drying without shade and open sun drying without shade was acceptable. And fenugreek dehydrated by open sun drying in shade was not acceptable even after two months of storage period

\section{Introduction}

Dehydration implies the partial removal of water from the agricultural product to a safe level of moisture content for preservation of quality, quantity, nutritional value and viability. Due to dehydration, concentration of solid increases leading to a reduction in water activity and the product becomes stable and free from insects and pests attacks, yeast and mould growth during further storage. Dehydration has also been practiced at domestic level by utilizing solar radiation. Solar drying can be considered as an 
elaboration to sun drying and is an efficient system of utilizing solar energy (Bala, 1997 and 1998, Zaman and Bala, 1998 and Muhlbauer, 1986). Open sun drying suffers from quality consideration though it enjoys cost advantage. This process has several disadvantages like spoilage of product due to adverse climatic conditions such as rain, wind, moisture and dust, loss of material due to birds and animals and deterioration of the material by decomposition, insects and fungus growth. This process is highly time consuming and require large area as well. Microwave drying is a relatively new addition in the existing drying techniques. With new and high microwave frequency, there are opportunities for new uses for microwave heating in the various drying processes such as corn, maize, unpolished rice as well as tobacco. Kaur (2001) reported the use of microwave for drying spices, garam masala and chilies.

Among the green leafy vegetables fenugreek (Trigonella foenum-graecum) is a popular green leafy vegetable in north India. It has excellent medicinal values. It is rich source of vitamin $A$, vitamin $C$, Iron, calcium and phosphorous and has an excellent aroma.

Dry leaves of fenugreek are used as herb. Being a seasonal crop, it is available in plenty at lower cost during winter season and therefore dehydrated to make it available in off season provided the quality of dehydrated product is maintained to be acceptable.

Fresh green leafy vegetables start to lose their quality immediately after harvest, becoming damaged, wilted and eventually rotten. Drying and storing fresh vegetables allows them to stay in good condition for a longer period. Drying fresh vegetables reduces bulkiness and weight and it became easy to store and transport, and avoids wastage. Storing and drying can provide human being a better diet year round.

\section{Materials and Methods}

\section{Preparation of samples}

Fresh Fenugreek (Trigonella foenumgraecum) was purchased from the local market. Before actual drying fresh fenugreek was sorted out and poor quality was rejected. Big stalk were removed and manual cleaning was done with the help of duster.

\section{Dehydration of samples}

The fenugreek was dehydrated by different methods, sun drying i.e. open sun drying without shade, open sun drying in shade, solar drying without shade, solar drying with shade, and microwave drying at two low power levels at $60 \%$ and $80 \%$.

\section{Storage of samples}

The dehydrated sample was packed in the airtight polythene bag of 200 gauge thickness and kept under room conditions for four months.

\section{Sensory evaluation of the dried fenugreek}

Physical property of dehydrated fenugreek was evaluated by panel of five judges immediately after dehydration and after two and four months of storage period in terms of colour, texture, flavour and overall appearance. The extent of acceptability was categorised as low (mean scores 1.0-1.7), medium (mean scores 1.8-3.4) and high (mean scores 3.5-5.0) on the basis of scores obtained.

The acceptability of the prepared product was tested by using dehydrated fenugreek by different methods. The recipe was tested by a panel of judges who were asked to score this for colour, flavour, texture, taste and overall acceptability. The extent of acceptability was categorised as low (mean scores1.0-1.7), 
medium (mean scores 1.8-3.4) and high (mean scores 3.5-5.0) on the basis of scores obtained. Prepared recipe was tested for two times for its acceptability after two and four months of storage period.

\section{Statistical analysis}

Mean scores were calculated for different parameters of quality of the product like colour, flavour, texture, taste and overall acceptability. One way analysis of variance was used to test the significant difference in the mean scores for various factors. Calculated values of variance ratio " $\mathrm{F}$ " compared with its corresponding table value at $5 \%$ level and Completely Randomised Design (CRD) technique was employed to calculate the critical difference at 5\% level of significance. The t-test was used to see the effect of storage period on selected recipes prepared with fenugreek dehydrated by different methods.

\section{Results and Discussion}

\section{Effect of storage period on overall acceptability scores of dehydrated fenugreek}

Physical property of dehydrated fenugreek was evaluated by panel of five judges immediately after dehydration and after two and four months of storage period in terms of colour, texture, flavour and overall appearance. The extent of acceptability was categorised as low (mean scores 1.0-1.7), medium (mean scores 1.8-3.4) and high (mean scores 3.5-5.0) on the basis of scores obtained.

It is clear from the Table 1 and Figure 1 that the overall acceptability of fenugreek dehydrated by open sun drying without shade decreased from $3.2 \pm 0.55$ to $2.8 \pm 0.84$ after two months but remained constant after two months. Fenugreek dehydrated by open sun drying had medium acceptability. There was no significant difference in overall acceptability after four months of storage period.

The overall acceptability of fenugreek dehydrated by open sun drying in shade also decreased from $1.6 \pm 0.55$ to $1.0 \pm 0.00$, after two months but remained constant after that. Fenugreek dehydrated by open sun drying in shade had low acceptability. There was significant influence of storage period on the overall acceptability of fenugreek.

The overall acceptability decreased from $3.6 \pm 0.55$ to $3.4 \pm 0.89$ for fenugreek dehydrated by solar drying without shade and from $3.8 \pm 0.45$ to $3.6 \pm 0.55$ for solar drying with shade after two months but it remained constant after two months. Fenugreek dehydrated by solar drying had high level of acceptability. But there was no significant difference in overall acceptability of both the samples.

In case of microwave drying at 60 per cent and 80 per cent power overall acceptability score remained constant up to two months. But from two months to four months it decreased from 4.0 to $3.8 \pm 1.1$ for 60 per cent and $4.2 \pm 84$ to 4.0 for 80 per cent power. Fenugreek dehydrated by microwave drying had high level of acceptability.

It was observed that mean score for overall acceptability decreased with the storage period but changes were not significant. From the analysis of variance it was inferred that storage period had no significant influence on the overall acceptability of fenugreek dehydrated by different methods except open sun drying in shade.

It was found that during the storage period of four months fenugreek dehydrated by microwave and solar drying with shade, solar drying without shade and open sun drying without shade was acceptable. 
Table.1 Effect of storage period on overall acceptability scores (Mean \pm SD) of dehydrated fenugreek

\begin{tabular}{|c|c|c|c|c|}
\hline Sample & $\begin{array}{l}\text { Immediately } \\
\text { After drying }\end{array}$ & After 2months & After 4 months & F-ratio \\
\hline$S_{1}$ & $3.2 \pm 0.55$ & $2.8 \pm 0.45$ & $2.8 \pm 0.84$ & $0.72^{\mathrm{NS}}$ \\
\hline $\mathbf{S}_{\mathbf{2}}$ & $1.6 \pm 0.55$ & $1.0 \pm 0.00$ & $1.0 \pm 0.00$ & $6.0^{*}$ \\
\hline $\mathbf{S}_{\mathbf{3}}$ & $3.6 \pm 0.55$ & $3.4 \pm 0.55$ & $3.4 \pm 0.89$ & $0.14^{\mathrm{NS}}$ \\
\hline $\mathbf{S}_{\mathbf{4}}$ & $3.8 \pm 0.45$ & $3.6 \pm 0.55$ & $3.6 \pm 0.55$ & $0.25^{\mathrm{NS}}$ \\
\hline $\mathbf{S}_{\mathbf{5}}$ & $4.0 \pm 0.71$ & $4.0 \pm 0.0$ & $3.8 \pm 1.1$ & $0.117^{\mathrm{NS}}$ \\
\hline$S_{6}$ & $4.2 \pm 0.84$ & $4.2 \pm 0.84$ & $4.0 \pm 0.0$ & $0.14^{\mathrm{NS}}$ \\
\hline \multicolumn{3}{|c|}{$\begin{array}{l}\text { "Significant at } 5 \text { percent level } \\
S_{1} \text { Open sun dried without sha }\end{array}$} & \multicolumn{2}{|c|}{ Non Significant } \\
\hline $\begin{array}{l}\mathrm{S}_{1} \\
\mathrm{~S}_{3}\end{array}$ & \multicolumn{2}{|c|}{$\begin{array}{l}\text { Open sun dried without sha } \\
\text { Solar dried without shade }\end{array}$} & $\begin{array}{l}\text { Open sun dried in } \\
\text { Solar dried with s }\end{array}$ & \\
\hline $\mathrm{S}_{5}$ & \multicolumn{2}{|c|}{ Microwave dried at $60 \%$ power } & \multicolumn{2}{|c|}{ Microwave dried at $80 \%$ power } \\
\hline
\end{tabular}

Fig.1 Effect of storage period on overall acceptability of dehydrated fenugreek
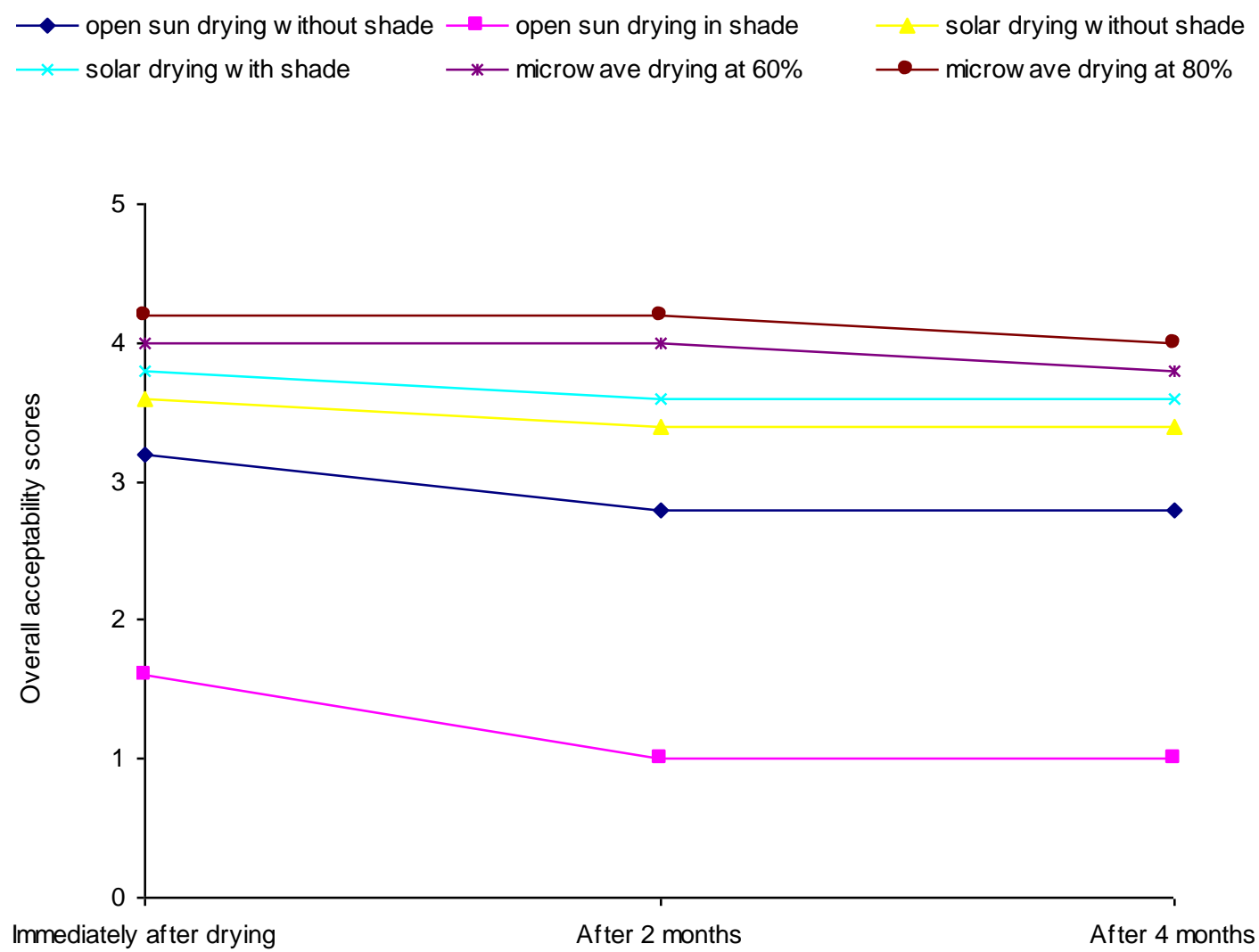

Storage period 
Table.2 Effect of storage period on overall acceptability score (Mean \pm SD) of sabji (Aloo-methi) prepared with fenugreek dehydrated by different methods after four months

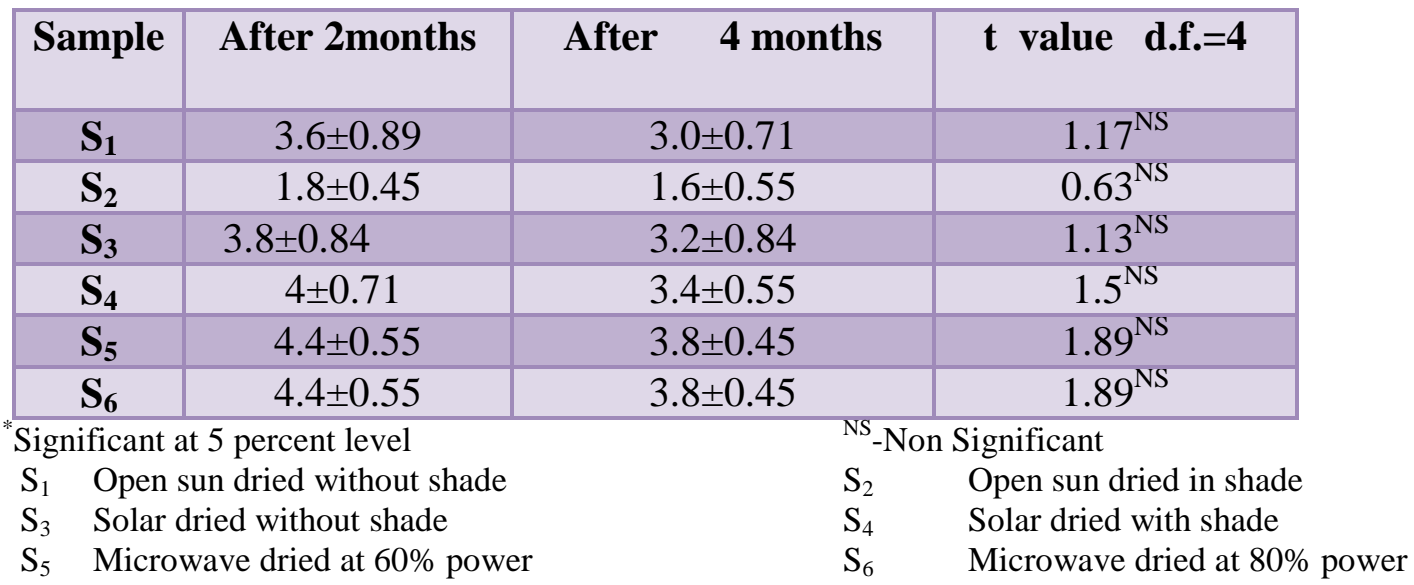

Fig.2 Effect of storage period on overall acceptability of sabji
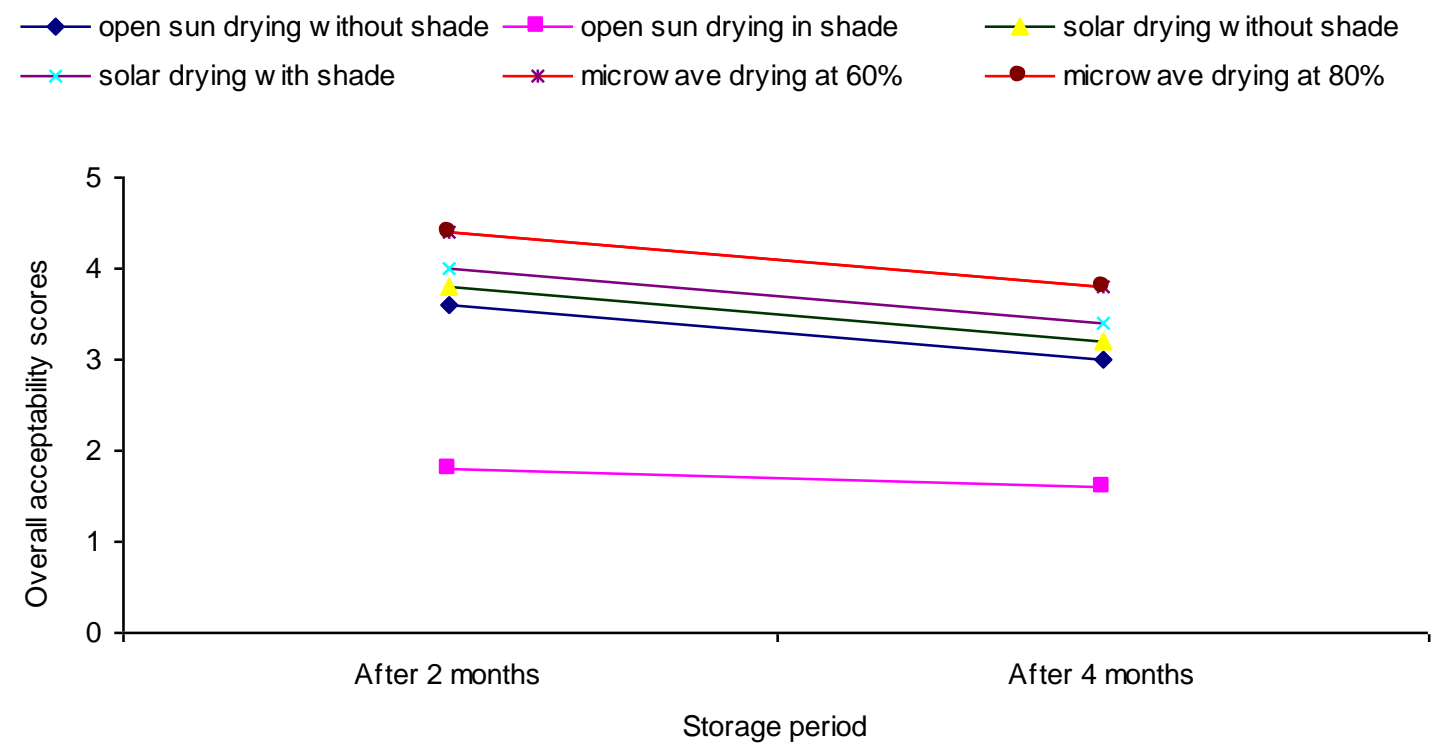

And fenugreek dehydrated by open sun drying in shade was not acceptable even after two months of storage period.

Effect of storage period on overall acceptability of sabji (Aloo-methi) prepared with fenugreek dehydrated by different methods after four months

It is clear from the Table 2 and Figure 2 that the overall acceptability of sabji (Aloo-methi) prepared with fenugreek dehydrated by open sun drying without shade and open sun drying with shade decreased from $3.6 \pm 0.89$ to $3.0 \pm 0.71$ and from $1.8 \pm 0.45$ to $1.6 \pm 0.55$ respectively from two months to four months. But there was no significant difference in overall acceptability.

The overall acceptability of sabji (Aloo-methi) prepared with fenugreek dehydrated by solar drying without shade and solar drying with shade decreased from $3.8 \pm 0.84$ to $3.2 \pm 0.84$ and from $4 \pm 0.71$ to $3.4 \pm 0.55$ respectively from 
two months to four months. But there was no significant difference in overall acceptability. The overall acceptability of sabji (Aloo-methi) prepared with fenugreek dehydrated by microwave drying at 60 power and $80 \%$ power decreased from $4.4 \pm 0.55$ to $3.8 \pm 0.45$ from two months to four months. But there was no significant difference in overall acceptability.

The overall acceptability scores decreased for sabji (Aloo-methi) prepared with fenugreek dehydrated by different methods after four months.

The overall acceptability scores of sabji prepared with fenugreek dehydrated by different methods decreased with storage period. Sabji prepared with fenugreek dehydrated by open sun drying in shade exhibited little change in overall acceptability scores. But there was no significant effect of storage period on overall acceptability score of recipe prepared by fenugreek dehydrated using different methods.

Fathima et al., (2001) also reported in their study that storage of fenugreek up to 60 days did not affect the colour, appearance, texture, taste and overall quality of the roti prepared with microwave dried fenugreek. On the whole it was concluded that overall acceptability decreased with the storage period but changes were not significant. Storage period had no significant influence on the overall acceptability of fenugreek dehydrated by different methods except open sun drying in shade.

It was found that during the storage period of four months fenugreek dehydrated by microwave and solar drying with shade, solar drying without shade and open sun drying without shade was acceptable. And fenugreek dehydrated by open sun drying in shade was not acceptable even after two months of storage period.

\section{References}

Bala, B.K. 1998. Solar drying system. Agrotech Publishing academy, India.

Fathima, A. Begum, K. and Rajalakshmi. 2001. Microwave drying of selected greens and their sensory characteristics. Plant Food for Human Nutr 56: 303-311.

Kaur, S. 2001. Evaluation of selected brands of microwave ovens for Indian cooking. M.Sc. thesis, Punjab Agricultural University, Ludhiana, India.

Muhlbauer, W. 1986. Present status of solar crop drying. Energy in Agriculture 5:121-137.

\section{How to cite this article:}

Janki Joshi and Jatinderjit K. Gill. 2019. Effect of Storage Period on Fenugreek Foliage Dehydrated by Different Methods. Int.J.Curr.Microbiol.App.Sci. 8(11): 764-769. doi: https://doi.org/10.20546/ijcmas.2019.811.090 